\title{
For the Love of Artifice 2: Attachment.
}

\author{
Dr. Trudy Barber
}

\section{An extension of the paper 'For the Love of Artifice: Why we need robot sex dolls and why there is a growing sub culture of real people trying to become them.'}

\author{
School of media and Performing Arts, Faculty of Creative and Cultural Industries, University \\ of Portsmouth, PO1 2DJ, UK. \\ Trudy.Barber@port.ac.uk
}

\begin{abstract}
This work is an extension to the original paper written for the AISB 50 Conference Symposium by the author 'For the Love of Artifice: why we need robot sex dolls and why there is a growing subculture of people trying to become them' where 'evolutionary sexual strategies according to Buss and Schmidt were introduced as a lens for discussing relations with artificial humans. In addition, this paper has combined Money's discussion of love mapping with Schwartz and White's theoretical approaches to attachment as a framework to explore our individual sexual strategies with artificial partners. It is argued in this extended paper that such theoretical approaches should be combined to facilitate discourse on the impact of robotic and technological intimacy on the end user. This need not necessarily be solely seen in terms of psychological aspects, but also in relation to Jenkins' notion of contemporary participatory culture, associations with multi-mediated geek fandoms and fetishes, and concepts of social acceptance. As a consequence of this, elements of attachment explored as a sexual project rather than an emotional one, as in erotic countertransference onto robots and technology for example, will be developed.
\end{abstract}

Keywords: Attachment, Cybersex, Intimacy, Geeks, Fetish, Love maps, Nerds, Sex robots, Sexual strategies, Technology.

\section{Introduction}

According to Ferguson [1], the contemporary sex doll or "fully functioning feminized android [ ... ] appears to have arrived at the threshold of the boundary between pleasure and science." ${ }^{1}$ As a consequence then, this paper will not re-visit popular associated discussions of misogyny, surrealist representations of the female

1 (Ferguson, 2010, p.3) 
form, ${ }^{2}$ nor the female robot through science fiction and fantasy narrative [2] - but will explore in a similar fashion as to what has been argued [3] as the "spectre of necrophilia, [a] lens through which the sexualisation of artificial humans has been viewed." ${ }^{3}$ In this light, this paper wishes to contrast the notion of making the artificial human as a sex toy - and it's associations with a deconstruction of what this paper terms the datafication of pleasure and, in this instance, the geekification of the end user - with what could be described by Buss and Schmidt [4] as the 'lens'of an evolutionary 'Sexual Strategies Theory', combined with the construction of a psychological 'Love Map' as argued by Money [5] together with 'Attachment Theory' as contextualized by Schwarz and White [6]. This combination will be used as a framework for exploring the construction of, and sexual engagement with, artifice.

\section{Contemporary Context}

For some, the idea of solitary engagement with high-tech artifice consolidates elements of intimate relations and possible emotional ties. Khan et al [7] explain "that to understand deep parts of human-robot interaction - and of what it means to be a human - we need to assess the possibilities and limits of psychological intimacy with robots." ${ }^{4}$ This in-turn reveals the potential capabilities of depth (or lack of it) of human to human intimacy. As suggested in the previous version of this paper - if this can be explored in terms of love and sex, we could also use artificial humans to understand sadism, cruelty, fear and violence. In which case would creating the features of hatred, anger, and sexual sadism for example be further challenging in the context of lengthy discussions surrounding the Uncanny Valley [8]? Hanson has argued that the "identification of fundamental principles of robot aesthetics can greatly accelerate the successful deployment of robots."[9] So there needs to be specific fundamental principles that would be needed to develop sexual interaction and engagement in which contemporary robots would be categorised - similar to other forms of entertainment such as movie or TV genres. The artificial robot created for sexual entertainment then, through a process of datafication of its various programmable responses and behaviors, can be branded and launched as a consumer product aimed at specific sections of market preference. The robot doesn't just need to overcome our reactions to the Uncanny Valley, but also needs to satisfy a more sophisticated and, according to Jenkins, a transmediated ${ }^{5}$ contemporary clientele who are

2 Bellmer, Hans. 1902 - 1975. For a general overview of his work see: https://en.wikipedia.org/wiki/Hans_Bellmer

3 (De Fren, 2009, p.409)

4 (Khan et al. 2010, p.124)

5 For a definition and further discussion on transmedia see: http://henryjenkins.org/2011/08/defining_transmedia_further_re.html 
used to having their specific inclinations and predilections catered for through narrative world-building and audience participation as enthusiasts and through fandom activities.[10]

\subsection{Popular Culture.}

The popularity of engagement with technological artifice such as robots ad sex dolls was discussed in the earlier version of this paper. To re-cap, it was argued that in contemporary narcissistic behaviors online (such as the 'selfie' for example) the concept of artifice, the robot and sex doll take on sexual and sub-cultural significance. This can be seen as evidence of forms of psychological transference which will be discussed in more detail in section 3. Whereas gender identity has been argued extensively by Butler as something that is performative [11], there are other performed identities that include notions of the robot and sex doll which are freely exposed on the Internet. This is a sub-cultural fan base of androidism: those who wish to perform as and appear to be robots and dolls. Venus Angelic from the UK is one such participant with an extensive transmedia presence - Venus Youtube Videos explain how to get the 'android look' ${ }^{6}$. Using a technique redolent of the popular BBC 3 TV youth programming reality TV 'make-under show' Snog Marry Avoid ${ }^{7}$, ironically Venus in the 'step by step' video - demonstrates her 'make-over' transformation into an android doll. The video states 'for the ANDROID look you only need to wear ONE scleral lens.' The process demonstrated is hypnotic, invasive and appears to feed back into notions of the Uncanny Valley once more. So popular culture then, has a particular role to play when we consider our interaction with the idea of the sex doll/robot. This is just one simple example of how our experiences of the robot in popular culture can color responses and attitudes. This when combined with a sexual strategy theory create an explosive mix which could influence and affect the design and creation of artifice for pleasure. There are responses of revulsion, humor, inquisitiveness and even disbelief that such desires exist for sexual interaction with the artificial human or robot. However, it has also been contested that robots for sexual intimacy need not necessarily take on human form or likeness, ${ }^{8}$ which could enable an even more innovative approach to creating inventions of pleasure. It is therefore argued that such creative approaches to pleasure are intrinsic to human nature, and should be included in articulations of 'evolutionary sexual strategy theory'.

6 Venus Angelic: How to look like an android doll http://www.youtube.com/watch?v=NU5NzchNkng

7 Snog, Marry Avoid. (2008 - ) BBC 3. Remarkable Television. UK.

8 Devlin, K (2016), Keynote Address. Second International Congress on Love and Sex with Robots. Goldsmiths University. London. UK. http://loveandsexwithrobots.org/ 


\section{Evolutionary Sexual Strategy.}

'A key ingredient of Sexual Strategies Theory is that mating strategies are context dependant, and in particular highly sensitive to the temporal context of short-term versus long-term mateships. ${ }^{99}$ In this quotation, Buss and Schmidt begin to lay out their Sexual Strategies Theory [4]. Should this viewpoint be applied to a sexual strategy of 'mateship' with artificial humans, the appreciation between long term commitments, versus short-term sexual release is revealed. This appreciation can vary from the type of investment made for the specificities of artificial interaction both emotionally and financially; and also reveals the context dependant on such participation with artificial humans. Buss and Schmitt postulate that 'long term mating, like all sexual strategies, carries costs when contrasted with alternative strategies. ${ }^{, 10}$ Evolutionary psychologists such as Buller [12] contest elements of such discussion and tend to argue around notions of homogamy and assortative mating mainly from a heterosexual standpoint and suggest that 'people tend to mate with those similar in race or ethnic background, age, socioeconomic status, educational background, and religious orientation. ${ }^{, 11}$ If this is applied to our relationship with technology and artificial humans and robots then, this also reveals something further about how our perceptions of intimacy have developed over chronological time and technological design time.

There are some interesting metaphorically descriptive discussions surrounding our psychology and subsequent interactions with technology and robots. The reliance of the socially interactive robot as being founded on deception has been considered as oversimplified by de Graaf [13]. However, in the past there have also been psychological descriptions of the human individual as a robot which is thought to be exhibited as part of narcissistic disorder and a representation of a 'pseudo-ego' [14]. There are also definitions of an automaton self and an automaton state in which an individual self-harms because of a lack of mutual reciprocity from others [15]. Inherent dangers of what is perceived as the unidirectional bond of the human with the robot have also been explored [16]. Much of these associations are seen as part of the general discourse of psychological disorders with possible social stigma and fear attached. It is therefore of no surprise that there appears to be a counter transference of such fears and stigma from society onto those who would have intimacy with robots and other technologies. On a personal level, the individual who may well engage in some form of transference with their chosen technology of pleasure are also engaging and investing in their own sexual strategy. In an empirical study by Scheutz and Arnold [17] it was argued that there is tension between technology and society when it comes to robot sex. They state:

9 (Buss and Schmidt, 1993, p205)

10 (Buss and Schmidt, 1993, p216)

11 (Buller, 2005, p213) 
"The tension in technology between social distancing and achieved intimacy requires closer empirical study into exactly what conditions and contexts make autonomous systems more harmful than helpful. This is especially important for the design and use of robots in sexual contexts, given the intricate and powerful norms, expectations, and associations that sex carries with it."12

Our understanding of a socially embedded technological otherness is now articulated as part of our very sense of self and how we engage with our intimately and selectively mediated construction of identity needs further exploration. Part of that construction and exploration, and our sense of attachment both sexual and romantic, it is argued, also involves the process of love mapping. This would also apply to building our relationship with technology and interaction with robots.

\section{$4 \quad$ Love Mapping}

According to Money [5] a "Lovemap"; is sexologically described as "a developmental representation or template of the mind and in the brain depicting the idealized lover and idealized program of sexuoerotic activity projected in imagery or actually engaged in with that lover." ${ }^{13}$ It is argued that this constant search for the creation of an individual lovemap is how and why contemporary and emerging digital media are used, as lovemapping provides a tool with which to sublimate many forms of deviant, experimental or usual sexual practices, relationship tinkering, or to explore identity formation and gender.

In light of our current relationships to the artificial, it would appear that connections and attachments to technology is an amplification of a long standing and traditional argument surrounding deviancy that Money has discussed in detail. Downing [18] reviews this and suggests that Money, through his hypothetical constructionist theory surrounding lovemaps and the genesis of paraphilias, argues that this is evidence of "social developments that have gone awry", which it is argued, is different from more traditional "nineteenth-century understanding of nature gone awry." ${ }^{\prime 14}$ It can therefore be suggested that the argument against the love of robots and our attachment to technology in general can be compounded by the concept of 'nature' against 'social developments' that deviate from tradition or what is considered a 'normative' understanding of human relationships - which in itself is contested. Lovemapping has been discussed by sexologists Benestad, Almå and Weingarten as the creation of "turn-on patterns." ${ }^{15}$ They have discovered that

12 (Scheutz and Arnold, 2016, p.358)

13 (Money, 1986, p.290)

14 (Downing, 2010, pp277-278)

15 (Benestad, Almå and Weingarten, 2015, p.27) 
through the exploration of lovemapping individuals have been able to identify and enjoy their turn-on patterns and find positive and ethical ways of practicing them [19]. This sex-positive approach would better contextualize the concept of lovemapping that includes relationships with robots. An example of this would be the engagement of a woman to her robot in hopes of marriage in the future. Not only does this challenge notions of human to robot relationships but also ethical issues, as well as the enactment of a more traditional lovemap involving heterosexual commitment. ${ }^{16}$

So the concept of 'lovemapping' in this context of humans doing so with robots, can be argued in terms of a manifestation of deviated social development, which this paper argues, can lead to even more original approaches to innovation and technological development. In our 'desire to be wired' there is also a revelation that openly displays our need to be connected. The examples in this paper explain how deviant sexual practices instigated by our push to find our individual sexual strategy and our love map extend the boundaries of technological development and emerging media and ethical engagement. However, it is not solely the technological hardware that needs to be developed it is also the content of such mediated behavior that inspires attachment that needs to be considered.

\section{$5 \quad$ Attachment}

Schwarz and White [6] argue that "attachment is seen as a source of human motivation as fundamental as those of food and sex." ${ }^{\text {"17 }}$ They go on to contest Bowlby's [20] postulation "that attachment behavior is any form of behavior that results in a person attaining or maintaining proximity to some other preferred and differentiated individual."18 They suggest that "sexuality was not a focus of theoretical concern for Bowlby" and that "he emphasized that attachment was a motivational system in its own right, apart from sexuality and feeding." ${ }^{\prime 19}$ Like other relational theories, Schwarz and White argue that attachment theory has been criticized for its failure to theorize sexuality adequately in light that "it has come to be understood that attachment is a bodily experience,20. They cite Mitchell [21] as evidence here, and argue that "within contemporary relational theories, sexuality has come to be seen as the central arena in which the dramas of attachment are played out- in which "emotional connection and intimacy is sought, established, lost and regained"21." ${ }^{22}$

${ }^{16}$ See: http://futureofsex.net/robots/lilly-inmoovator-engaged-human-robot-couple-want-rightmarry/

17 (Schwarz and White, 2005, p.7)

18 (Bowlby, 1979, p. 129)

19 (Schwarz and White, 2005, p.7)

20 (Ibid.)

21 (Mitchell, 1988, p. 107). 
It is also suggested therefore, that intimacy and attachment to a robot or technological other for example, adds a new dimension to what had been discussed and theorized as 'adult attachment styles' by Hudson-Allez [22]. Such articulations should be considered as value potential for integrative theoretical models for advancing relationship science, as well as providing insight into attachment related behaviors such "as safe-haven and secure-based functions", ${ }^{23}$ similar to those of a human attachment figure.[23]

Dewitte's [24] review on sex, attachment and human to human relationships, argues that it is important to focus on "the processes that mediate the link between sex and attachment”24. Dewitte confirms that part of the process of focus within this research is to explore an emotion-motivational model in combination with evolutionary and attachment perspectives. She states that "the emotion-motivational perspective specifies the different processes and pathways through which attachment schemas influence sexual responses."25 By conceptualizing the attachment and the sexual system in terms of emotion regulation Dewitte suggests would set up new and alternative lines of inquiry into sex and attachment. If this approach to research is translated directly to sexual activity between humans, sex robots and artificial humans, it may prove invaluable as to how to explore the process of attachment that we generate with our technologies of pleasure. This may also be able to explain whether we feel that there are potential processes that could also be translated from a transverse viewpoint; that of whether robots can become attached to humans. Robots could then not only feel attachment to humans, but would also have the potential to be attached to each other.

\section{Summary.}

This paper has brought together approaches from different disciplines in order to engage with concepts of robot sex. It has argued that through the use of robots, stimulation and communication technology or artificial humans for sexual interaction, an individual can test out their own issues surrounding attachment and intimacy. This is inclusive of the continuous recreation, re-affirmation and performance or 'acting out' of a psychological love map that induces attachment to the object or device concerned as part of an individual and personalized sexual strategy. However, due to various portrayals of robots in popular culture, sex with robots can be seen as a manifestation of a deviant form of social development that some may find awkward and associated with counter transference and stigma. This is all too easily

22 (Schwarz and White, 2005, p.7)

23 (Birnbaum et al, 2016, p.417)

24 (Dewitte, 2012, p.119)

25 (Dewitte, 2012, p.120) 
associated with another conceptual context articulating that such behavior is 'against nature' and part of the Frankenstein approach to technology, in that it has the potential to be 'out of control' and therefore dangerous. There is a feminist movement - The Campaign Against Sex Robots - that aims to ban sex and technological activities along with anthropomorphic and animistic articulations which are redolent of radical Dworkinite fears and the demeaning of sex workers in general and women in particular. However, it is argued that this can also be seen a contemporary example of deviation as key to innovation [25] and as a blatant opportunity to explore sexuality and the human condition in even more depth in a sex-positive way that reveals more about our need to be creative, innovative and inventive as part of our human evolutionary sexual strategy as a whole. In conclusion, it is hoped that the development of robots and artificial humans that may be able to respond to us and to each other will contribute to the evolution of our sense of sexual self and may eventually break the notion of a feared unidirectional approach to relationships, to emerging technologies and eventually, to love.

\section{References.}

[1] Ferguson, A. (2010) The Sex Doll: A History. Mc Farland Publishing.

[2] Barber, T. (2009) 'Kinky Borgs and Sexy Robots: the fetish, fashion and discipline of Seven of Nine'. In Geraghty L. (Ed) Future Visions: Key Science Fiction and Fantasy Television Texts. Scarecrow Press. Pp $133-148$.

[3] de Fren, A. (2009) Technofetishism and the Uncanny Desires of A.S.F.R. (alt.sex.fetish.robots). Science Fiction Studies, Vol. 36, No. 3, pp. 404-440. SF-TH Inc.

[4] Buss, D. Schmitt, D. (1993) Sexual Strategies Theory: An Evolutionary Perspective on Human Mating. Psychological Review Vol. 100. No. 2. 204-232.

[5] Money, J. (1986) Lovemaps: Clinical concepts of sexual/erotic health and pathology, paraphilia and gender transposition in childhood, adolescence and maturity. New York: Irvington.

[6] Schwartz, J., and White, K., (Eds.) (2005) Sexuality and Attachment in Clinical Practice. London, GB: Karnac Books.

[7] Kahn, P.H. Jr., Ruckert J.H., Kanda, T., Ishiguro, H., Reichert, A., Gary, H., Shen, S., (2010) Psychological Intimacy with Robots? Using interaction patterns to uncover depth of relation. Proceedings of the $5^{\text {th }}$ ICM/IEEE International Conference on Human - Robot Interaction.

[8] Mori, M (1970) The Uncanny Valley. Energy, 7(4), pp. 33-35 
[9] Hanson, D. (2006). Exploring the aesthetic range for humanoid robots. In Proceedings of the ICCS/CogSci-2006 Symposium: Toward Social Mechanisms of Android Science, Vancouver, Canada.

[10] Jenkins H (2006) Convergence Culture: Where Old and New Media Collide. NYU Press.

[11] Butler, J. (1990) Gender Trouble: Feminism and the Subversion of Identity. Routledge. London.

[12] Buller, D. J. (2005) Adapting Minds: Evolutionary Psychology and the Persistent Quest for Human Nature. MIT Press.

[13] Maartje M. A. de Graaf (2016) An Ethical Evaluation of Human-Robot Relationships International. Journal of Social Robotics, August, Volume 8, Issue 4, pp 589-598

[14] Ledermann, R., (1981) The Robot Personality in Narcissistic Disorder Journal of Analytical Psychology (26) 329-344.

[15] Sweet, A. D. (2011) The Automaton Self: Defensive organisation, psychodynamics and treatment approaches. Psychodynamic Practice Vol. 17, No. 4, November, 387-402

[16] Scheutz M (2012) The inherent dangers of unidirectional emotional bonds between humans and socially interactive robots. In: Lin P, Abney K, Bekey GA (eds) Robot ethics: the ethical and social implications of robotics. MIT Press, Cambridge

[17] Scheutz M., and Arnold, T. (2016) Are We Ready for Sex Robots? Human Robot Interaction (HRI) $11^{\text {th }}$ ACM/IEEE International Conference.

[18] Downing, L (2010) John Money’s 'Normophilia’: diagnosing sexual normality in late-twentieth-century Anglo-American Sexology. Psychology \& Sexuality. Vol 1, No. 3. September, pp 275-287

[19] Benestad, E.E.P., Almå, E., and Weingarten, K. (2015) Sex-positive ways of perceiving sex turn-on patterns. Part 1: Understanding. International Journal of Narrative Therapy and Community Work. No 1.

[20] Bowlby, J. (1979). The Making and Breaking of Affectional Bonds. London: Tavistock.

[21] Mitchell, S. A. (1988). Sex without drive (theory). In: S.A. Mitchell (Ed.), Relational Concepts in Psychoanalysis. Cambridge, MA: Harvard University Press. 
[22] Hudson-Allez, G. (2010) Infant Losses; Adult Searches: A Neural and Developmental Perspective on Psychopathology and Sexual Offending. Karnac Books. London

[23] Birnbaum, G.E., Mizrahi, M., Hoffman, G.,Reis H. T., Finkel, Eli J., Sass, O., (2016) What Robots can teach us about intimacy: The reassuring effects of robot responsiveness to human disclosure. Computers in Human Behavior (63) 416-423.

[24] Dewitte, M. 2012 Different Perspectives on the Sex-Attachment Link: Towards an Emotion-Motivational Account. Journal of Sex Research, 49(2-3), 105-124.

[25] Barber, T. (2004) Deviation as Key to Innovation: understanding a culture of the future. Foresight Vol.6 No.3. 141 -152. 